\title{
Short Circuit Synchronous Electric Charge Extraction (SC-SECE) Strategy for Wideband Vibration Energy Harvesting
}

\author{
A Morel, P Gasnier, Y Wanderoild, G Pillonnet \\ Univ. Grenoble Alpes, F-38000 Grenoble, France \\ CEA, LETI, MINATEC, F-38054 Grenoble, France \\ adrien.morel@cea.fr
}

\author{
A Badel \\ Univ. Savoie Mont Blanc, SYMME, \\ F-74000 Annecy, France
}

\begin{abstract}
This paper presents an energy harvesting strategy called Short Circuit Synchronous Electric Charge Extraction (SCSECE), which is an improvement of the SECE strategy for highly coupled piezoelectric generators. The SC-SECE strategy is based on the control of a short-circuit through two tuning parameters. The first one, $\phi_{S}$, represents the phase between the displacement extrema and the energy harvesting event. The second, $\Delta \phi$, is the angle difference between the beginning and end of the short-circuit phase. The performance of this strategy has been compared to other interfaces such as the SECE and the capacitance tuning approach. However, with the SC-SECE strategy, both the power and bandwidth are greatly improved. Experimental measurements have been performed on a highly-coupled piezoelectric harvester $\left(\mathrm{k}_{\mathrm{m}}{ }^{2}=0.48\right)$ which provides a maximal harvestable power $P_{\text {lim }}$ of $6 \mu \mathrm{W}$ under an extremely low vibration amplitude $(0.03 G)$. We have been able to harvest more than $50 \%$ of this $P_{\lim }$ over a large frequency band, from $90 \mathrm{~Hz}$ to $140 \mathrm{~Hz}$. These results demonstrate the excellent potential of this strategy for extending the bandwidth of piezoelectric vibration energy harvesters.
\end{abstract}

Keywords - Vibration Energy Harvesting, Multiphysics modelling, Piezoelectricity, Interface circuit, Short-circuit control, SECE.

\section{INTRODUCTION}

Over the last two decades, scavenging ambient energy has been investigated as a possible replacement of or complement to batteries in order to make small systems and sensors autonomous [1]. Piezoelectric energy harvesting is particularly interesting in closed and confined environments where temperature gradients and solar light are insufficient to supply a sensor. One of the main building blocks of a piezoelectric energy harvester $(\mathrm{PEH})$ is the interface circuit which has to optimize the extraction of the energy stored in the piezoelectric material. Several synchronous strategies have been proposed, exhibiting high performances for lowly-coupled piezoelectric harvesters [2-5]. However, for highly-coupled PEH's, those strategies are no longer optimal since they overdamp the mechanical resonator $[2,6]$. In order to match the damping induced by the electrical interface with the mechanical one and enhance the energy extraction process for highlycoupled systems, new strategies have recently been proposed $[6,7]$. Some of these even induce a tuning of the resonant frequency over a certain frequency range $[8,9]$. However, we propose a new strategy called SC-SECE based on a short-circuit tunable phase which allows optimization of the harvested energy while improving the frequency tuning range compared to other state-of-the-art interfaces. Instead of accumulating the energy in the piezoelectric material during a whole semi-period as in the SECE approach, the harvester is short-circuited during a portion of the vibration semi-period. This then decreases the damping induced by the electrical circuit, and also leads to a change in the electromechanical system resonant frequency. In this paper, we first propose an analysis of the effects of a short circuit. Then, we analytically prove that our technique, when associated with highly coupled piezoelectric generators, greatly improves both the harvested energy and the bandwidth compared to the classical SECE strategy. Finally, we validate the theoretical analysis through experimental measurements on a highly-coupled PEH connected to an interface circuit implementing the proposed strategy.

\section{MODELING OF THE SC-SECE STRATEGY}

\section{A. PEH modeling}

A linear PEH is usually composed of a piezoelectric material deposited on a cantilever beam. Applying Newton's law to this mechanical system, we can find the dynamic equation of the mass displacement, $x$. From the fundamental equations for piezoelectric devices, we can then find the differential equation linking the derivative of the displacement, $\dot{x}$, the piezoelectric voltage, $v_{p}$, and the current extracted in the interface circuit, $i$. Finally, the displacement is considered periodic with a constant amplitude $X_{m}$. These three equations are summarized by the system (1).

$$
\left\{\begin{array}{c}
M \ddot{x}+D \dot{x}+K_{s c} x+\alpha v_{p}=-\mathrm{F}=-M \ddot{y} \\
i=\alpha \dot{x}-C_{p} v_{p} \\
x(t)=X_{m} \cos (\theta)=X_{m} \cos (\omega t)
\end{array}\right.
$$

where $y$ and $F$ are the ambient displacement and vibration force applied on the whole system, respectively. $M$ is the dynamic mass of the system, $K_{S C}$ the short-circuited stiffness, $D$ the mechanical damping of the structure, $C_{p}$ the piezoelectric clamped capacitance and, finally, $\alpha$ is the electromechanical 
coupling between the mechanical part and electrical part of the piezoelectric generator. These equations can be modeled as an equivalent electrical circuit as shown in Fig.1.

\section{B. Expression of the piezoelectric voltage}

In order to express the harvested power at every extraction event, we need to solve (1) and find the displacement amplitude, $X_{m}$. To do so, we first have to find the expression of the piezoelectric voltage $v_{p}$. The piezoelectric element is either working in an open-circuit or short-circuit condition (Fig.2), and hence its voltage expression over a time period can be given by (2).

$$
\mathrm{v}_{\mathrm{p}}(\theta)=\left\{\begin{array}{c}
\left.\left.\frac{\alpha}{\mathrm{C}_{\mathrm{p}}} \int_{\phi_{\mathrm{s}}+\Delta \phi-\pi}^{\theta} \dot{x}(\theta) \mathrm{d} \theta, \forall \theta \in\right] \phi_{\mathrm{s}}+\Delta \phi-\pi, \phi_{\mathrm{s}}\right] \\
\left.0, \forall \theta \in] \phi_{\mathrm{s}}, \phi_{\mathrm{s}}+\Delta \phi\right] \\
\left.\left.\frac{\alpha}{\mathrm{C}_{\mathrm{p}}} \int_{\phi_{\mathrm{s}}+\Delta \phi}^{\theta} \dot{x}(\theta) \mathrm{d} \theta, \forall \theta \in\right] \phi_{\mathrm{s}}+\Delta \phi, \phi_{\mathrm{s}}+\pi\right] \\
\left.0, \forall \theta \in] \phi_{\mathrm{s}}+\pi, \phi_{\mathrm{s}}+\Delta \phi+\pi\right]
\end{array}\right.
$$

$\phi_{\mathrm{s}} \in[0, \pi]$ is the phase between the energy harvesting event and the previous displacement extremum, whereas $\Delta \phi \in$ $[0, \pi]$ represents the angular time spent in the short circuit condition during a semi-period of vibration. A system implementing this extraction strategy is depicted in Fig.1, and an example of the voltage waveform is shown in Fig.2. As given by (2), $v_{p}$ is non-sinusoidal. In order to analytically solve (1) while simplifying the calculations, only the first harmonic of $v_{p}$ is considered. Using Fourier series, its expression can be obtained and is given by (3).

$$
\left\{\begin{array}{l}
\left.\mathrm{v}_{\mathrm{p}}(\theta)\right|_{\mathrm{n}=1}=\mathrm{a}_{1} \cos (\theta)+\mathrm{b}_{1} \sin (\theta) \\
\mathrm{a}_{1}=\frac{2}{\pi} \int_{\phi_{\mathrm{s}}+\Delta \phi-\pi}^{\phi_{\mathrm{s}}+\Delta \phi} v_{p}(\theta) \cos (\theta) d \theta \\
\mathrm{b}_{1}=\frac{2}{\pi} \int_{\phi_{\mathrm{s}}+\Delta \phi-\pi}^{\phi_{\mathrm{s}}+\Delta \phi} v_{p}(\theta) \sin (\theta) d \theta
\end{array}\right.
$$

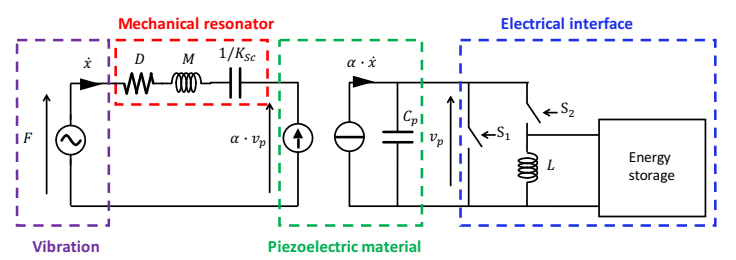

Fig. 1. PEH electrical model and system view of the SC-SECE strategy

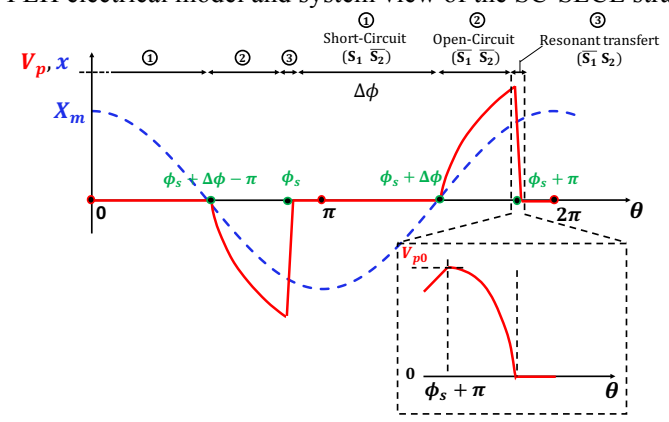

Fig. 2. Voltage and displacement waveforms of the SC-SECE strategy.

From equations (1), (2) and (3), we obtain the expression of the first Fourier series coefficients, given by (4).

$$
\left\{\begin{array}{c}
\mathrm{a}_{1}=\frac{\alpha \mathrm{X}_{\mathrm{m}}}{\pi \mathrm{C}_{\mathrm{p}}}\left[\pi-\Delta \phi+\frac{\sin \left(2 \phi_{s}+2 \Delta \phi\right)}{2}+\frac{\sin \left(2 \phi_{s}\right)}{2}\right. \\
\left.+2 \cos \left(\phi_{s}+\Delta \phi\right) \sin \left(\phi_{s}\right)\right] \\
\mathrm{b}_{1}=-\frac{\alpha \mathrm{X}_{\mathrm{m}}}{\pi \mathrm{C}_{\mathrm{p}}}\left[\cos \phi_{S}+\cos \left(\phi_{S}+\Delta \phi\right)\right]^{2}
\end{array}\right.
$$

Thus, the first harmonic of the piezoelectric voltage can be expressed in the Fourier domain as:

$$
\underline{\mathrm{v}_{\mathrm{p}_{1}}}=\underline{\mathrm{x}}\left(\frac{\mathrm{a}_{1}}{X_{m}}-\mathrm{j} \frac{\mathrm{b}_{1}}{X_{m}}\right)=\underline{\mathrm{x}}\left(\mathrm{a}_{1}^{*}-\mathrm{j} \mathrm{b}_{1}^{*}\right)
$$

Where $\underline{x}$ is the displacement written in the Fourier domain, and $a_{1}^{*}$ and $b_{1}^{*}$ are the first Fourier coefficients $a_{1}$ and $b_{1}$ divided by the displacement amplitude, $X_{m}$.

\section{Expression of the harvested power}

Considering that only the first voltage harmonic has an impact on the mechanical system dynamic due to the filtering effect of the resonator, we can substitute the voltage expression (5) in (1) in order to find the displacement amplitude. Solving the differential equation in the Fourier domain, we eventually obtain the displacement amplitude, given by (6).

$$
\left\{\begin{array}{c}
X_{m}=\frac{F}{\sqrt{A^{2}+B^{2}}} \\
A=K_{s c}-M \omega^{2}+\alpha \mathrm{a}_{1}^{*} \\
B=\omega D+\alpha \mathrm{b}_{1}^{*}
\end{array}\right.
$$

For every semi-period, the harvested energy is that stored in the piezoelectric capacitance $C_{p}$ when $\theta=\phi_{\mathrm{s}}$. Thus, from (1) and (2), the general harvested power expression can be given as a function of the displacement amplitude, as shown in (7).

$$
\mathrm{P}=\frac{\omega \alpha^{2}}{2 \pi C_{p}} \mathrm{X}_{\mathrm{m}}^{2}\left[\cos \left(\phi_{s}+\Delta \phi\right)+\cos \left(\phi_{s}\right)\right]^{2}
$$

From (4), (6) and (7), we can now determine the harvested power for any parameter couple $\left(\phi_{s}, \Delta \phi\right)$.

\section{MODEL ANALYSIS AND PERFORMANCE ESTIMATION}

The energy harvested with a particular extraction strategy is dependent on the PEH used, and varies with the product $k_{m}^{2} Q_{m}$, where $k_{m}^{2}$ is the modified squared coupling coefficient, and $Q_{m}$ the mechanical resonator quality factor $[2,10]$. It also varies with the normalized vibration frequency, $\Omega_{m}$, which is the ratio of the angular vibration frequency, $\omega$, to the angular mechanical resonant frequency of the $\mathrm{PEH}, \omega_{0}$. We introduced the normalized extractable power $\mathrm{P} / \mathrm{P}_{\text {lim }}$ of the standard SECE in Fig. 3, where $\mathrm{P}_{\lim }$ is the maximum harvestable power as defined in [10]. Fig. 4 shows the same analysis for the capacitive tuning approach, which consists in adapting the resistive and 
capacitive load of a PEH in order to tune its stiffness, as extensively explained in [9]. Finally, the results of the proposed SC-SECE approach are depicted in Fig.5.

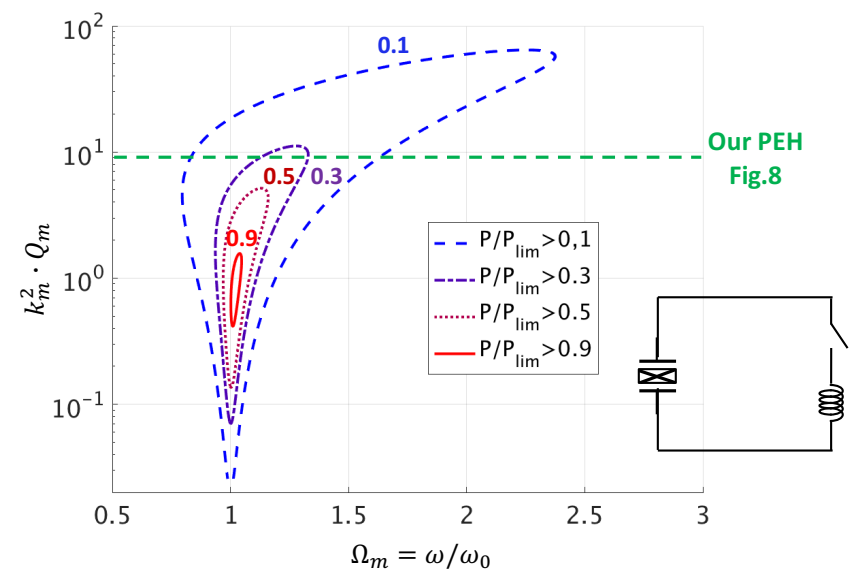

Fig. 3. Normalized analysis of the harvested power with a standard SECE interface [2-4] in the $k_{m}^{2} Q_{m}-\Omega_{m}$ plane, where $Q_{m}=20$.

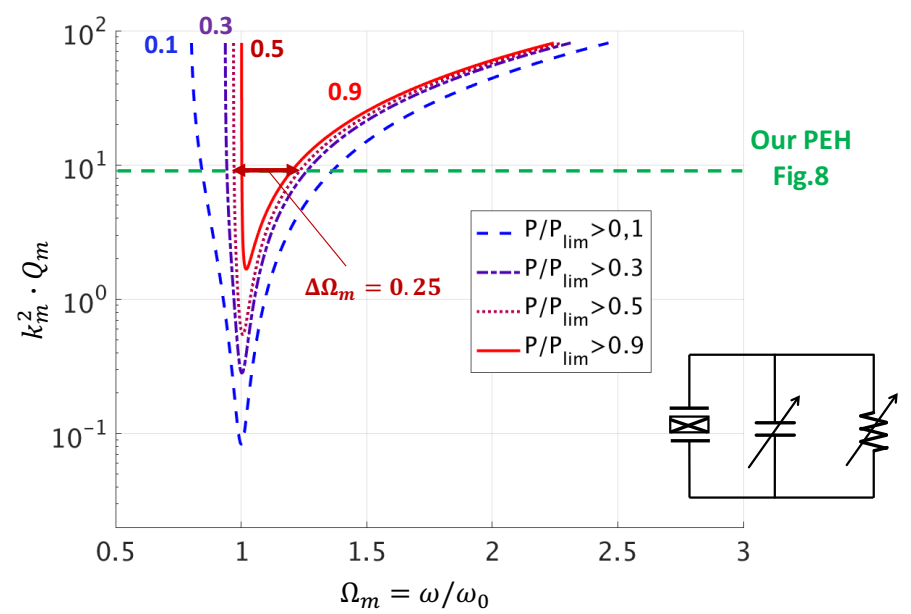

Fig. 4. Normalized analysis of the harvested power with a capacitive tuning interface [9] in the $k_{m}^{2} Q_{m}-\Omega_{m}$ plane where $Q_{m}=20$.

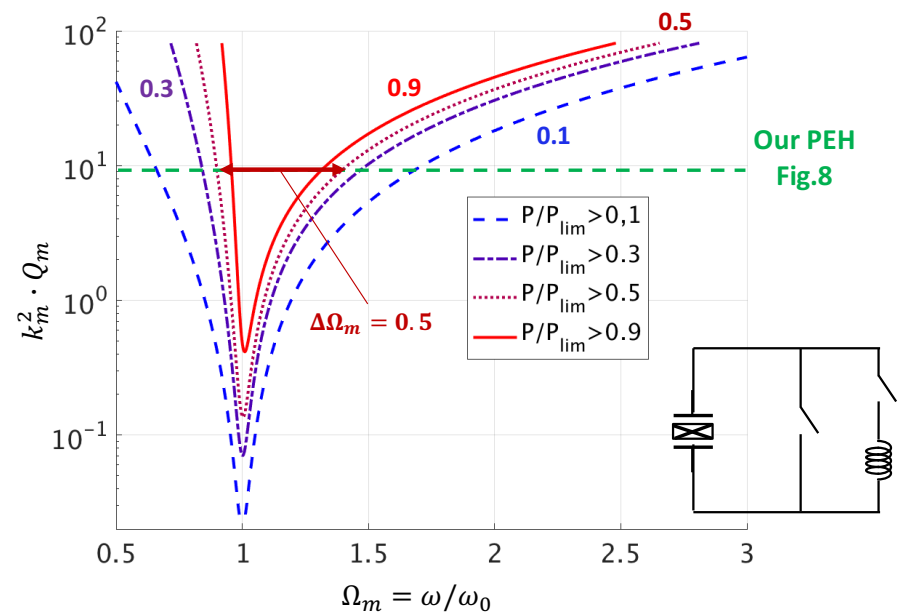

Fig. 5. Normalized analysis of the harvested power thanks to the proposed SC-SECE interface in the $k_{m}^{2} Q_{m}-\Omega_{m}$ plane where $Q_{m}=20$.
We can verify from Fig.3 that the SECE interface is only fully efficient when both the product $k_{m}^{2} Q_{m}$ is about $\pi / 4$ and $\Omega_{m}$ is about 1 [2]. Thus, this strategy is sensitive to both external conditions (vibration frequency) and the intrinsic characteristics of the PEH $\left(k_{m}^{2}\right.$ and $\left.Q_{m}\right)$. The capacitive tuning strategy improves the robustness of the system with respect to the vibration frequency. However, this approach requires the use of a bulky capacitive bank. Finally, Fig. 5 shows that the proposed SC-SECE strategy greatly enhances the frequency tuning range compared to other approaches, while continuing to provide a maximal power close to the power limit $\mathrm{P}_{\text {lim }}$ of any PEH.

In order to evaluate the SC-SECE performance, a new FoM is established (8). This represents the integral of the power frequency response with one strategy compared to that of an optimized resistive load. The maximum harvestable power as well as the FoM are shown on Figures 6 and 7 for the different strategies. For lowly-coupled/highly-damped PEHs, the SECE and SC-SECE are the most effective. When $k_{m}^{2} Q_{m}$ is higher than $\pi / 4$, the maximum harvested power with the SECE strategy starts decreasing while the two other strategies reach $\mathrm{P}_{\text {lim }}$ for $k_{m}^{2} Q_{m} \geq \pi$ for the capacitive tuning and for $k_{m}^{2} Q_{m} \geq$ $\pi / 4$ for the SC-SECE strategies. For any $k_{m}^{2} Q_{m}$, the SC-SECE exhibits a higher FoM than any other strategies.

$$
F O M=\frac{\int_{0}^{\infty} P_{\text {Strategy }}(\omega) d \omega}{\int_{0}^{\infty} P_{d c}(\omega) d \omega}
$$

Fig. 6. Maximum harvestable power as a function of $k_{m}^{2} Q_{m}$ with the standard SECE interface (blue), the capacitive tuning approach (purple), and the proposed SC-SECE (red).

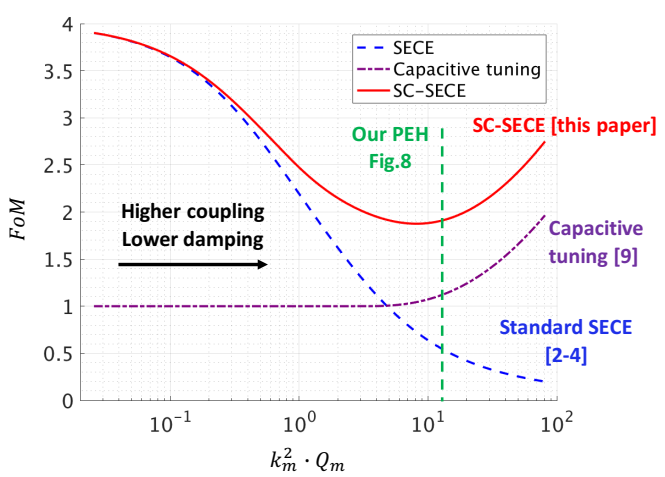

Fig. 7. FoM comparison of the three strategies as a function of $k_{m}^{2} Q_{m}$ 


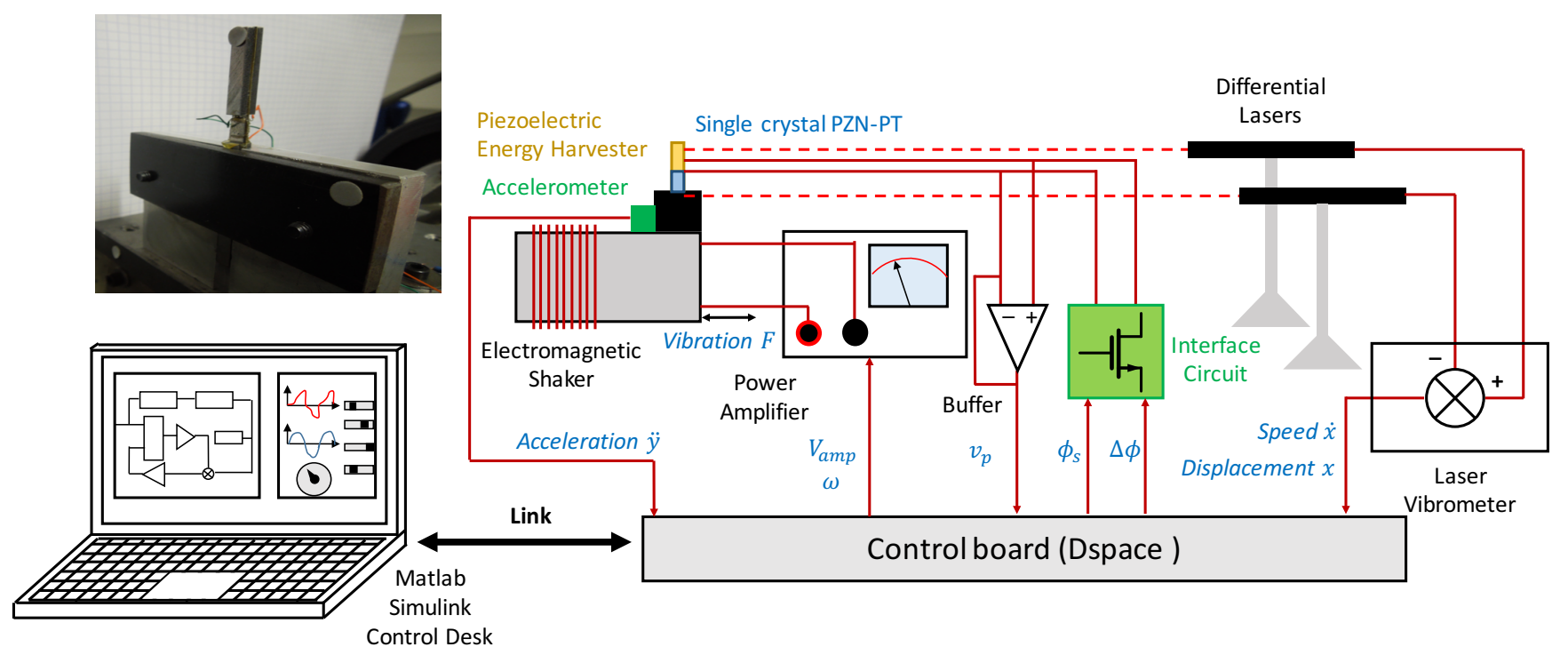

Fig. 8. Experimental setup with automated data collection. The piezoelectric generator is made of $10 \mathrm{x} 5 \mathrm{x} 0.5 \mathrm{~mm}^{3}$ plates of PZN-PT single crystals which have been manufactured by Microfine Company.

\section{EXPERIMENTAL VALIDATION}

\section{A. Experimental setup}

The experimental setup is presented in Fig.8. A highlycoupled piezoelectric generator is fixed on an electromagnetic shaker which vibrates at an angular frequency $\omega$ and an amplitude $F_{m}$. The mass displacement $x$ and speed $\dot{x}$ are sensed by differential lasers. The piezoelectric voltage waveforms are both memorized and controlled using a Dspace control board dynamically fixing the parameters $\phi_{S}$ and $\Delta \phi$. In our experiment, we have collected data for $\left(\phi_{S}, \Delta \phi, \omega\right) \in[0, \pi] \times$ $[0, \pi] \times[565,943]$, recording precisely $30 \times 30 \times 100$ sets of waveforms.

\section{B. Piezoelectric generator characteristics}

The PEH shown in Fig. 8 has been characterized: its shortcircuit angular frequency $\omega_{0}=628 \mathrm{rad} . \mathrm{s}^{-1}$, squared coupling coefficient $k_{m}^{2}=0.48$, and quality factor $Q_{m}=20$. The piezoelectric capacitance $C_{p}$ is $1.5 \mathrm{nF}$. The tests have been performed for a vibration amplitude of $0.03 \mathrm{G}$, and the harvested power limit $\mathrm{P}_{\text {lim }}$ of our PEH under this vibration is $6 \mu \mathrm{W}$.

\section{Measurement results}

The measurement results are presented in Fig.9. The collected data allowed us to plot the maximal harvested power as a function of the vibration frequency, as well as the optimal couples $\left(\phi_{S}, \Delta \phi\right)$ for any vibration frequency. These results can be compared to the theoretical ones which have been obtained by solving (7) with the PEH parameters. As shown in Fig. 9, the experimental results are in good agreement with the model. We observed both theoretically and experimentally, that for some frequencies, two optimal couples $\left(\phi_{S}, \Delta \phi\right)$ which maximize the harvested power can be found. Combined with our highlycoupled PEH, the SC-SECE strategy allowed us to harvest more than $50 \%$ of the maximum harvestable power $\mathrm{P}_{\text {lim }}$ on a relative frequency range $\Delta \omega / \omega_{0}$ as large as $50 \%$, from $90 \mathrm{~Hz}$ to $140 \mathrm{~Hz}$, which is more than any other reported energy harvesting strategy.

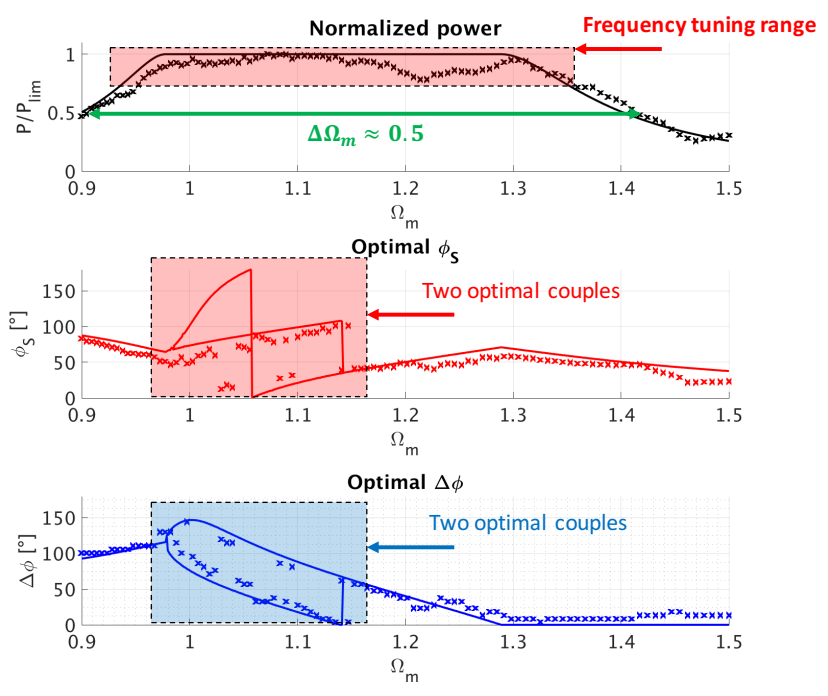

Fig. 9. Experimental (dots) and theoretical (plain lines) maximum harvested power results and optimal $\left(\phi_{S}, \Delta \phi\right)$.

\section{CONCLUSION}

In this paper, we propose a new extraction strategy called SC-SECE. By adjusting both the energy harvesting event phase, and the length of a short circuit, this strategy allows the harvested energy to be optimized while tuning the system's electromechanical resonant frequency over a large frequency range. For our PEH (Fig.8), the proposed SC-SECE increases the harvestable energy bandwidth by about $200 \%$ compared to a previously proposed capacitive-based tunable interface [9]. In order to implement this strategy in an adaptive interface, the main remaining challenge is to design an algorithm which dynamically determines the couples $\left(\phi_{S}, \Delta \phi\right)$ which maximize the harvested power. This algorithm, if it successfully combines fast convergence and low power consumption, would lead to an efficient way to tune the resonant frequency of linear piezoelectric harvesters. 


\section{REFERENCES}

[1] J. Krikke, "Sunrise for energy harvesting products" IEEE Pervasive Comput., vol. 4, issue 1, 2005.

[2] E. Lefeuvre et al., "A comparison between several vibration-powered piezoelectric generators for standalone systems", Sensors and Actuators A: Physical, vol. 126, no. 2, pp. 405-416, Feb. 2006.

[3] A. Quelen et al., "A 30nA Quiescent $80 \mathrm{nW}$ to $14 \mathrm{~mW}$ Power Range Shock-Optimized SECE-based Piezoelectric Harvesting Interface with $420 \%$ Harvested Energy Improvement”, IEEE International Solid State Circuit Conference, to be published.

[4] T. Hehn et al., "A Fully Autonomous Integrated Interface Circuit for Piezoelectric Harvesters", IEEE Journal of Solid-State Circuits, vol. 47, no. 9, pp. 2185-2198, Sep. 2012.

[5] G. A. Rincon-Mora and S. Yang, "Tiny piezoelectric harvesters: Principles, constraints and power conversion", IEEE Trans. Circuits Syst. I, Reg. Papers, vol. 63, no. 5, pp. 639-649, May 2016.

[6] A. Morel et al., "Regenerative synchronous electrical charge extraction for highly coupled piezoelectric generators", IEEE Midwest symposium of circuits and systems (MWSCAS) 2017, 2017.

[7] A. Richter et al., "Tunable interface for piezoelectric energy harvesting", in 2014 IEEE 11th International Multi-Conference on Systems, Signals Devices (SSD14), pp. 1-5, 2014.

[8] A. Morel et al., "Active AC/DC control for wideband piezoelectric energy harvesting," Journal of Physics: Conference Series. 773, 012059, 2016.

[9] B. Ahmed-Seddik et al., "Self-powered resonant frequency tuning for piezoelectric vibration energy harvesters", Journal of Physics: Conference Series 476, 012069, 2013.

[10] A. Badel et al. "Nonlinear Conditioning Circuits for Piezoelectric Energy Harvesters", in: E. Blokhina, A.E. Aroudi, E. Alarcon, D. Galayko (Eds.), Nonlinearity Energy Harvest. Syst., Springer International Publishing, pp. 321-359, 2016. 